\title{
An unusual tumor spectrum in Lynch syndrome caused by MSH6 mutation
}

\author{
Renee L Perrier ${ }^{*}$, Paulein Van Galen, Janice L Pasieka, Tony Magliocco, A Micheil Innes \\ From 13th Annual Meeting of the Collaborative Group of the Americas on Inherited Colorectal Cancer \\ Honolulu, Hawaii, USA. 16-17 October 2009
}

\begin{abstract}
Background
Inherited cancer syndromes associated with acoustic neuroma (i.e. neurofibromatosis 2-NF2), pheochromocytoma (i.e. Von Hippel Lindau, NFl, multiple endocrine neoplasia syndromes, and hereditary paraganglioma syndrome), and colon cancer are well known. Lynch syndrome is the most common hereditary colon cancer syndrome and is caused by DNA mismatch repair dysfunction secondary to inherited mutations in one of MLHl, MSH2, MSH6, and less commonly PMS2. An increased risk for a variety of cancers is seen in patients with Lynch syndrome with the greatest risks being for colon and endometrial cancer. We report a Dutch patient with a history of bilateral acoustic neuromas diagnosed at 47 , and pheochromocytoma and endometrial adenocarcinoma diagnosed at age 54 . She had no family history or other signs/symptoms of NF2. Family history was significant for her brother having metachronous colon cancers at 42 and 51, and a maternal uncle having colon cancer in his 40s. The family does not fulfill either Amsterdam I or II criteria.
\end{abstract}

\section{Methods}

Clinical investigations for hereditary cancer predisposition were undertaken in our patient given her history of multiple primary tumors. Immunohistochemistry (IHC) for MLH1, MSH2, and MSH6 proteins, and genetic testing for Lynch syndrome were completed. In light of the history of bilateral acoustic neuromas, genetic testing for NF2 was also undertaken.

\section{Results}

Genetic testing for NF2 did not detect a mutation or deletion in the NF2 gene. IHC on tissue from the patient's endometrial adenocarcinoma and pheochromocytoma showed absent expression of MSH6. A pathogenic germline mutation in MSH6 (c.651_652insT) was identified.

\section{Conclusions}

We report a potential new association of Lynch syndrome with pheochromocytoma and acoustic neuromas in a woman with Lynch syndrome caused by an MSH6 mutation. In this case, a diagnosis of Lynch syndrome was suspected due the history of endometrial adenocarcinoma in our patient and her family history of early colon cancer. Identification of a Dutch founder mutation in MSH6 confirmed the diagnosis. The absence of MSH6 expression by IHC in both the endometrial carcinoma and the pheochromocytoma highly suggests an association of the pheochromocytoma with defective mismatch repair function secondary to the MSH6 mutation. Unfortunately, tumor studies could not be completed on tissue from the acoustic neuroma so it is difficult to say whether they arose independently or were also related to the diagnosis of Lynch syndrome. In this case, normal NF2 genetic testing and the absence of a family history of NF2 may suggest an association of acoustic neuromas with Lynch syndrome.

Published: 25 May 2010

doi:10.1186/1897-4287-8-S1-P17

Cite this article as: Perrier et al:: An unusual tumor spectrum in Lynch syndrome caused by MSH6 mutation. Hereditary Cancer in Clinical Practice 2010 8(Suppl 1):P17.

* Correspondence: renee.perrier@albertahealthservices.ca

Alberta Children's Hospital, Calgary, Alberta T3B 6A8, Canada 